13

\title{
Исследование динамики разогрева анодных узлов в безмасочном нанолитографе на основе массива микрофокусных рентгеновских трубок
}

\author{
(ㄱ П.Ю. Глаголев, Г.Д. Демин, Н.А. Дюжев, М.А. Махиборода, Н.А. Филиппов
}

Национальный исследовательский университет „МИЭТ“, 124498 Зеленоград, Москва, Россия

e-mail: skirdovf@mail.ru

Поступило в Редакцию 30 апреля 2021 г.

В окончательной редакции 30 апреля 2021 г.

Принято к публикации 30 апреля 2021 г.

Исследована динамика разогрева матрицы узлов анодной мембраны с прострельной мишенью под действием автоэмиссионного тока, генерируемого в электронной системе безмасочного рентгеновского нанолитографа. Определены перспективные материалы мембраны, обеспечивающие эффективный теплоотвод от матрицы анодных узлов, среди которых алмазоподобные пленки показали наилучший результат. При рассчитанной мощности мягкого рентгеновского излучения $P_{X}=2.5 \mathrm{nW}$, рассеиваемой на пикселе размером $20 \mathrm{~nm}$, и дозе облучения рентгенорезиста $D=100 \mathrm{~J} / \mathrm{m}^{2}$ время экспонирования составило $25 \mu \mathrm{s}$. Показано, что за время экспонирования пластины диаметром $150 \mathrm{~mm}$ алмазоподобная анодная мембрана размером $300 \times 300$ элементов разогревается от 20 до $62^{\circ} \mathrm{C}$, что в 21 раз ниже температуры разогрева альтернативного материала анода из Si. Описан технологический маршрут изготовления матрицы анодных узлов с учетом предложенных способов оптимизации ее конструкции, направленных на понижение тепловых эффектов разогрева при проведении процессов рентгеновской нанолитографии. Полученные результаты могут быть применимы при разработке системы микрофокусных рентгеновских трубок в составе безмасочного рентгеновского нанолитографа.

Ключевые слова: рентгеновская нанолитография, микрофокусная рентгеновская трубка, прострельная мишень, матрица анодных узлов, тепловой разогрев, термическое расширение, Bosch-процесс.

DOI: $10.21883 / J T F .2021 .10 .51372 .132-21$

\section{Введение}

В последние десять лет наблюдается стремительное возрождение вакуумной наноэлектроники, что главным образом связано с появлением технологических возможностей для реализации новых типов вакуумных устройств с длиной проводящего канала менее $100 \mathrm{~nm}$ [1-3]. Одним из приоритетных направлений вакуумной наноэлектроники является автоэмиссионная электроника, где на фоне большого количества фундаментальных и прорывных исследований в данной области [4-7] удалось достичь значительных практических результатов и реализовать высокотехнологичные электронные устройства на автоэмиссионном принципе действия (диоды $[8,9]$, нанотранзисторы [10,11], наносенсоры [12], портативные рентгеновские трубки [13] и т.д.). В связи с этим большой интерес для промышленного производства представляет возможность создания безмасочного нанолитографа на основе массива микрофокусных рентгеновских трубок.

На данный момент выделяют два основных вида безмасочных методов литографии для индустриальных применений - оптическая и электронная литографии [14,15] (рис. 1). Недостатком оптической безмасочной литографии является низкое разрешение, которое позволяет обеспечить формирование топологии рисунка с проектными нормами более $200 \mathrm{~nm}[16,17]$. Очевидно, что наиболее универсальным и продвинутым методом является электронная литография. Она удовлетворяет практически всем требованиям к гипотетическому безмасочному литографу, за исключением одного производительности, которая падает на шесть-семь по-

\section{Matrix of anode nodes}

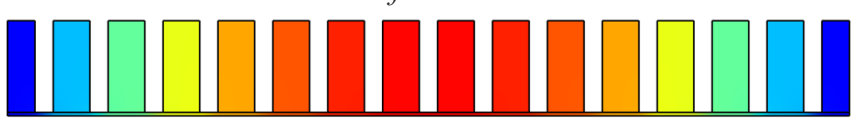

Matrix of field emission cathode-grid nodes

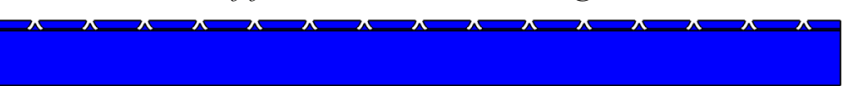

Рис. 1. Схематическое изображение системы источников мягкого рентгеновского излучения на основе матрицы АКУ и матрицы анодных узлов. Матрица АКУ состоит из набора кремниевых острийных катодов с управляющими сеточными электродами. Матрица анодных узлов в начальной конструкции состоит из бериллиевой прострельной мишени, расположенной на перфорированном электроде анода. Цветовая гамма приведена для отображения разогрева матрицы анодных узлов при воздействии на него электронного тока с матрицы АКУ. 
рядков с уменьшением топологии от микрометрового до нанометрового уровня [14]. Наибольшие перспективы связываются с многолучевой электронной литографией. Благодаря малой длине волны даже относительно медленных электронов потенциально эта технология обеспечивает фундаментальное ограничение разрешения на уровне $1 \mathrm{~nm}$. Основная проблема электронной литографии - это электро-магнитостатическое взаимодействие между нанопучками, приводящее к размытию изображения. Несмотря на то что многолучевой электронной литографией занимаются уже десятки лет, проблема до сих пор не решена. В частности, наилучшие результаты получены в компании Mapper Technology. Установка последнего поколения работает с 65 тыс. пучков и поддерживает технологические нормы $32 \mathrm{~nm}$. Размер элементарного пучка составляет $25 \mathrm{~nm}$, производительность до 4 пластин в час. Данные исследования выявили проблему сильного нагрева пластин и, как следствие, с ограничения разрешения и точности совмещения.

Поэтому исследования в области безмасочной рентгеновской литографии являются крайне актуальными для развития наноэлектроники следующего поколения. Причем это относится как к классической высокопроизводительной литографии в части выбора длины волны короче $13.5 \mathrm{~nm}$ и технологий создания многослойных зеркал с дифракционным качеством, так и для создания ключевых технологий безмасочной рентгеновской литографии.

Ранее в работах [18-20] нами была предложена концепция рентгеновского нанолитографа на основе массива микрофокусных рентгеновских трубок (МРТ). Технологически такой массив МРТ состоит из матрицы анодных узлов с прострельной мишенью, а также матрицы автоэмиссионных катодных узлов и матрицы отверстий управляющего (сеточного) электрода, самосовмещенных между собой и образующих матрицу автоэмиссионных катодно-сеточных узлов (АКУ). Основа одиночного АКУ - полупроводниковый катод острийного типа, предназначенный для генерации автоэмиссионного тока под действием вытягивающего напряжения анода и управляющего напряжения сеточного электрода, запирающего или открывающего соответствующий канал для эмиссии электронов. Одиночный анодный узел состоит из бериллиевой прострельной мишени и участка анодной мембраны (анодного электрода) с рентгеновским окном (отверстие в анодном узле, определяющее направленность рентгеновского излучения на выходе из прострельной мишени). Прострельная мишень предназначена для конверсии энергии электронов, поступающих с „открытого“ для протекания тока АКУ, в мягкое рентгеновское излучение. Анодный электрод с рентгеновским окном выполняет основные две функции функцию самосовмещения с прострельной мишенью и функцию генерации электрического поля между АКУ и прострельной мишенью в результате приложения к нему вытягивающего напряжения. Необходимо отметить, что матрицы АКУ и анодного узла самосовмещены и имеют размерность $300 \times 300$ элементов с шагом $3 \mu \mathrm{m}$.
В целях обеспечения надежности и воспроизводимости процессов рентгеновской нанолитографии особое внимание следует уделять стабильной работе как отдельных элементов МРТ, так и всей системы в целом. В предыдущей работе [21] нами обсуждались методы и способы повышения электростатической стабильности матрицы анодных узлов. Однако не менее важным фактором, который сказывается на работе всей рентгенооптической системы, является ее устойчивость к температурным воздействиям, поскольку бомбардировка прострельной мишени высокоэнергетичными электронами (с энергией до $2 \mathrm{keV}$ и выше) может вызывать серьезный разогрев матрицы анодных узлов. В результате это становится причиной заметной термической деформации матрицы анодных узлов, что негативным образом может сказаться на работе всего устройства [22]. В свою очередь, термическое расширение материала матрицы анодных узлов может привести к сужению диаметра рентгеновского окна в соответствующем единичном анодном узле и, следовательно, уменышению выходной мощности рентгеновского излучения в данной области. Таким образом, это может привести к росту времени набора соответствующей дозы рентгеновского излучения для экспонирования чувствительного рентгенорезиста. Кроме того, направленность рентгеновского излучения на выходе из отдельного анодного узла определяется пространственным положением стенок соответствующего рентгеновского окна. В процессе термической деформации матрицы анодных узлов может произойти провисание анодной мембраны, что приводит к отклонению от вертикального положения стенок рентгеновских окон. В результате изменяется направленность рентгеновского излучения на выходе из матрицы анодных узлов, что может привести к искажению и размытию топологического рисунка на пластине. К тому же разогрев анода до температуры выше критической величины (более $1400^{\circ} \mathrm{C}$ для $\mathrm{Si}$ ) может привести к необратимым последствиям - деградации и оплавлению всей конструкции или отдельных элементов матрицы анодных узлов.

Чтобы не допустить подобные нежелательные эффекты, необходимо рассмотреть возможные механизмы отвода тепла с матрицы анодных узлов. Очень часто отвод тепла в рентгеновских трубках осуществляется с помощью активной воздушной или жидкостной системы охлаждения, контактирующей с нагретым анодом [23]. Однако в случае рентгеновского нанолитографа подобная активная система охлаждения потребует усложнения конструкции самой рентгенооптической системы. По этой причине наиболее предпочтительным является пассивное охлаждение матрицы анодных узлов путем выбора оптимального состава материалов прострельной мишени и анодной мембраны, которые позволят обеспечить высокоэффективный теплоотвод с матрицы на остальную конструкцию нанолитографа. Для выполнения указанной задачи интересно рассмотреть различные типы материалов, обладающих высокой теплопроводностью $k$, среди которых в ра- 
боте нами были выбраны: $\mathrm{C}\left(k=2500 \mathrm{~W} \cdot \mathrm{m}^{-1} \cdot \mathrm{K}^{-1}\right)$, $\mathrm{Al}\left(k=237 \mathrm{~W} \cdot \mathrm{m}^{-1} \cdot \mathrm{K}^{-1}\right), \mathrm{Cu}\left(k=401 \mathrm{~W} \cdot \mathrm{m}^{-1} \cdot \mathrm{K}^{-1}\right)$, $\mathrm{Si}\left(k=130 \mathrm{~W} \cdot \mathrm{m}^{-1} \cdot \mathrm{K}^{-1}\right)$, Ni $\left(k=90.7 \mathrm{~W} \cdot \mathrm{m}^{-1} \cdot \mathrm{K}^{-1}\right)$ и $\mathrm{Fe}\left(k=80.2 \mathrm{~W} \cdot \mathrm{m}^{-1} \cdot \mathrm{K}^{-1}\right)$.

\section{1. Описание модели разогрева матрицы анодных узлов}

Для расчета разогрева матрицы анодных узлов под действием направленных электронных пучков с матрицы АКУ в программной среде COMSOL MultiPhysics на первом шаге была проведена оценка площади электронного пятна $S_{D}$ на анодной мембране на заданном расстоянии $d_{C A}=10 \mu \mathrm{m}$ между наконечниками острийных катодов и анодом (при напряжении на аноде $V_{A}=2 \mathrm{kV}$ и напряжении $V_{G}=30 \mathrm{~V}$ на сеточном электроде). Данный расчет проводился путем построения траекторий электронов с поверхности катода при заданном распределении электрического поля $\mathbf{E}(\mathbf{r})=-\nabla \varphi(\mathbf{r})$ в межэлектродном пространстве, что соответствует уравнениям:

$$
\begin{gathered}
\frac{d m_{e} \mathbf{v}(\mathbf{r})}{d t}=q_{e} \mathbf{E}(\mathbf{r}), \\
\nabla^{2} \varphi(\mathbf{r})=-\rho_{v} \varepsilon_{0},
\end{gathered}
$$

где $m_{e}, q_{e}$ - масса и заряд электрона, $\mathbf{v}(\mathbf{r})-$ скорость движения электронов, $\varphi(\mathbf{r})$ - распределение потенциала в межэлектродном пространстве, $\varepsilon_{0}$ - диэлектрическая постоянная, $\rho_{v}-$ плотность заряда. Результаты моделирования показали, что радиус электронного пятна на мишени составляет $R \approx 350 \mathrm{~nm}$. Распределение плотности электронов по пятну не равномерно и может быть описано функцией Гаусса (соответствует нормальному распределению). Поскольку в пределах окружности, ограничивающей 50\% площади пятна, находится около 90\% электронов, вводится эффективный радиус $R_{\mathrm{eff}}=R / \sqrt{2}$, а эффективная площадь электронного пятна оценивается как $S_{D}=\pi R_{\mathrm{eff}}^{2} \approx 0.196 \mu \mathrm{m}^{2}$. С учетом мощности электронного пучка $P_{e}$, приходящей на одиночный анодный узел, и суммарной площади $S_{D}^{\Sigma}=\sum_{i=1 \ldots n}$ участков мембраны, на которую воздействует ток по открытым для эмиссии $n$ каналам, была определена суммарная рассеиваемая мощность электронных пучков $Q_{s}=P_{e}^{\Sigma} / S_{D}^{\Sigma}$, участвующая в разогреве конструкции матрицы анодных узлов, где $P_{e}^{\Sigma}=\sum_{i=1 \ldots n} P_{e}^{i}$. Далее был произведен численный расчет температуры разогрева анодной мембраны с заданной конструкцией, материалами и составом слоев, для чего применялось уравнение теплопроводности, записанное в общем виде как:

$$
\rho C_{p}\left(\frac{\partial T}{\partial t}+\mathbf{u}_{\text {trans }} \nabla T\right)+\nabla\left(\mathbf{q}+\mathbf{q}_{\mathbf{r}}\right)=Q_{S},
$$

где $\rho-$ плотность $\left[\mathrm{kg} / \mathrm{m}^{3}\right] ; C_{p}-$ удельная теплоемкость при постоянном давлении $[\mathrm{J} /(\mathrm{kg} \cdot \mathrm{K})] ; T-$ абсолютная температура $[\mathrm{K}]$; $\mathbf{u}_{\text {trans }}$ - вектор скорости теплопередачи $[\mathrm{m} / \mathrm{s}] ; \mathbf{q}=-k \nabla T-$ вектор конвективного теплового потока $\left[\mathrm{W} / \mathrm{m}^{2}\right], k$ - теплопроводность $[\mathrm{W} / \mathrm{m} \cdot \mathrm{K}] ; \mathbf{q}_{\mathbf{r}}$ вектор теплового потока за счет излучения $\left[\mathrm{W} / \mathrm{m}^{2}\right]$.

Расчет времени экспонирования отдельного участка рентгенорезиста позволяет оценить скорость работы разрабатываемого нанолитографа и время экспонирования всей пластины целиком. Следует отметить, что предварительная оценка времени экспонирования отдельного участка рентгенорезиста необходима для моделирования разогрева матрицы анодных узлов под воздействием электронного тока с матрицы АКУ в течение данного времени. Время экспонирования $t_{\text {ехр }}$ отдельного участка (пикселя) рентгенорезиста можно оценить как:

$$
t_{\exp }=D / P_{S}
$$

где $D$ - доза облучения чувствительного рентгенорезиста и $P_{S}$ - рассеиваемая мощность рентгеновского излучения на пикселе, которую можно рассчитать, зная мощность рентгеновского излучения $P_{X}$ и площадь одного пикселя $S_{p i x}$ :

$$
P_{S}=P_{X} / S_{p i x}
$$

Расчет мощности рентгеновского излучения $P_{X}$, падающего на рентгенорезист, проводился согласно уравнению из [24]:

$$
P_{X}=P_{e} C_{E} R_{m}^{2} \Omega_{i n} / 4 \pi .
$$

где $C_{E}$ - коэффициент конверсии мощности электронов $P_{e}$ в мощность линии рентгеновского излучения, $R_{m}$ - представляет собой коэффициент отражения рентгеновских зеркал Мо/Ве двухзеркального объектива, рассмотренных в [25], $\Omega_{\text {in }}$ - телесный угол, в пределах которого излучение источника падает на объектив (полезное излучение источника).

\section{2. Оценка времени экспонирования рентгенорезиста}

С помощью уравнений (4)-(6) оценено время экспонирования $t_{\exp }$ отдельного участка рентгенорезиста. Коэффициент конверсии для бериллиевой пленки толщиной $200 \mathrm{~nm}$ и энергии электронов $2 \mathrm{kV}$ составляет $C_{E}=2.5 \cdot 10^{-4}[24]$, мощность электронов $P_{e}=2 \cdot 10^{-5} \mathrm{~W}$, телесный угол $\Omega_{\text {in }}=2 \pi$, коэффициент отражения многослойных рентгеновских зеркал Мо/Be в спектральном диапазоне рентгеновского излучения (в окрестности $11.4 \mathrm{~nm}) R_{m}=0.7$ [26]. Мощность электронного пучка с одиночного анодного узла можно рассчитать, зная электронный ток с отдельного острийного катода $I_{\text {single }}=10 \mathrm{nA}$ и напряжение между электродом анода и матрицей АКУ $V_{C A}=2 \mathrm{kV}$ по формуле $P_{e}=I_{\text {single }} V_{C A}$. Подставив все переменные в уравнение (6), можно рассчитать мощность рентгеновского излучения, равную $P_{X}=1.25 \cdot 10^{-9} \mathrm{~W}$. Согласно [27], 
$a$
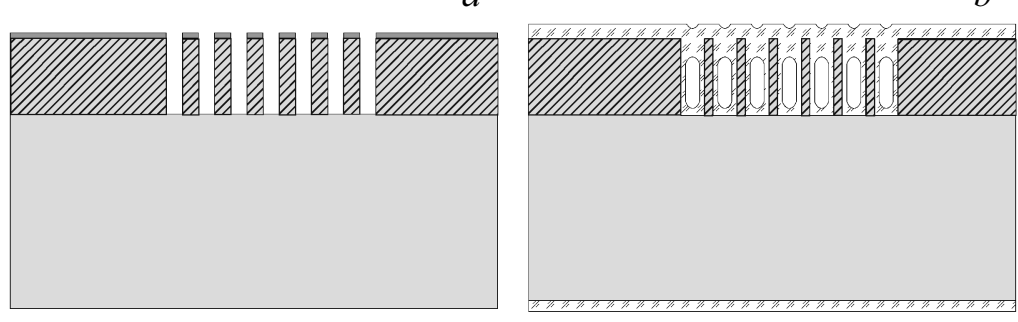

$b$
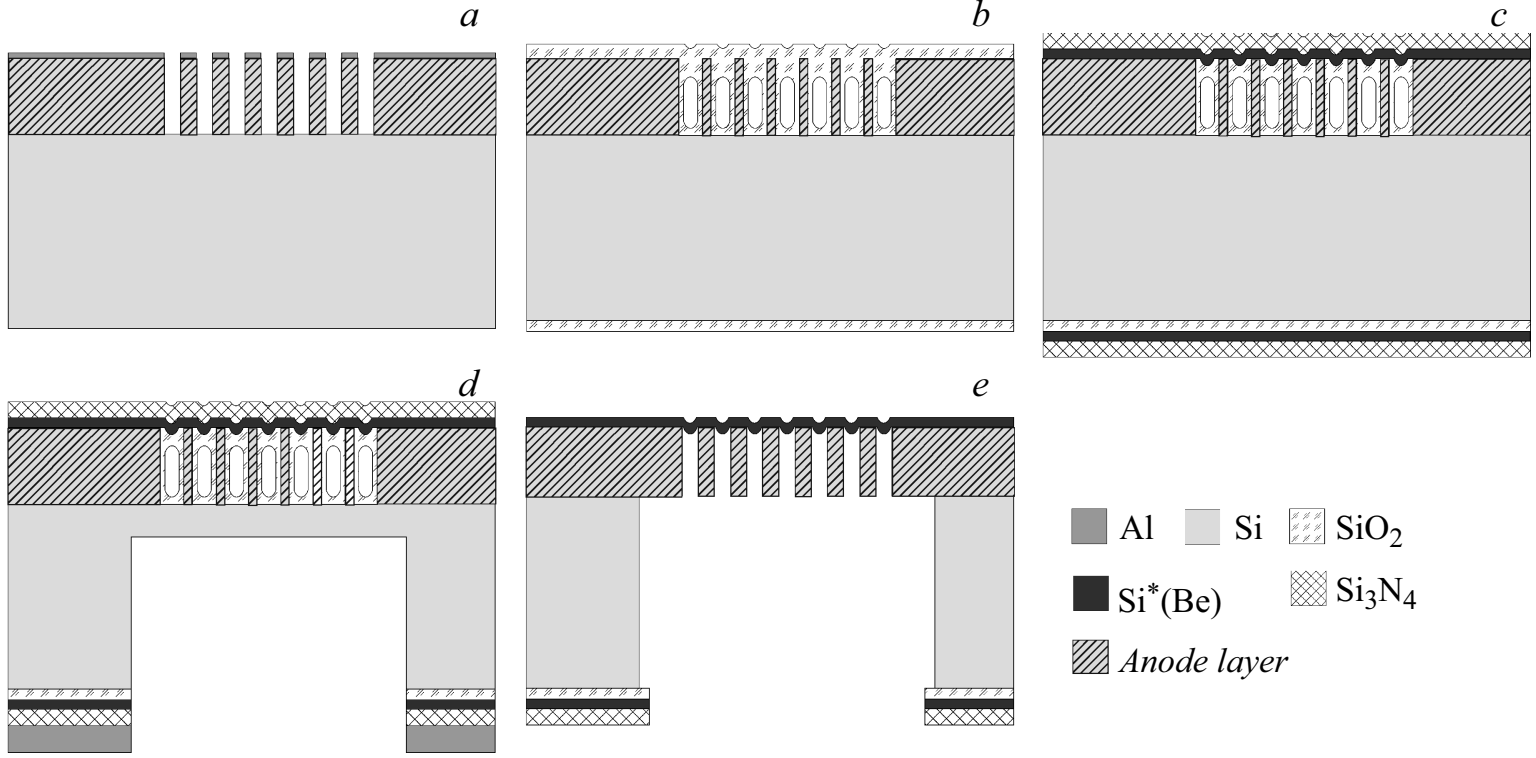

Рис. 2. Технологические этапы изготовления матрицы анодных узлов с прострельной мишенью: $a-$ Bosch-процесс по маске Al (реактивное ионное травление); $b-$ оплавление борофосфороселикатного стекла (БФСС); $c-$ осаждение $\mathrm{Si}_{3} \mathrm{~N}_{4} ; d-\mathrm{Bosch}$ процесс с обратной стороны пластины с небольшим недотравом; $e-$ вытравливание БФСС и $\mathrm{SiO}_{2}$ из каналов в парах НF.

диаметр пикселя разрабатываемого литографа составляет $20 \mathrm{~nm}$, отсюда рассеиваемая мощность рентгеновского излучения на пикселе, согласно уравнению (5), будет равна

$$
P_{S}=1.25 \cdot 10^{-9} /\left(\pi\left(10^{-8}\right)^{2}\right) \approx 0.4 \cdot 10^{7} \mathrm{~W} / \mathrm{m}^{2}
$$

Поскольку доза облучения для чувствительного рентгенорезиста составляет $D=10^{2} \mathrm{~J} / \mathrm{m}^{2}$ [27], тогда время экспонирования $-t_{\exp }=25 \mu \mathrm{s}$. Следует отметить, что разрабатываемый микрофокусный литограф рассчитан на матрицу $300 \times 300$ элементов, поэтому способен проявлять одновременно участок рентгенорезиста площадью $36 \mu \mathrm{m}^{2}$.

\section{3. Технологические этапы изготовления матрицы анодных узлов с прострельной мишенью}

В случае выбора кремния (Si) в качестве материала анода при изготовлении матрицы анодных узлов производится Bosch-процесс травления Si на глубину, равную толщине электрода анода, по маске $\mathrm{Al}$ (рис. 2,a). Затем стравливается Al и проводится окисление на $100 \mathrm{~nm}$ (это делается для того, чтобы щелочной травитель не попадал внутрь каналов и не растравливал кремний). В случае если необходимо сделать электрод анода из алмазоподобной пленки $(C)$, производится процесс ее осаждения на кремниевую подложку. Далее с помощью реактивного ионного травления вытравливаются каналы под рентгеновские окна на всю толщину электрода анода. Необходимо отметить, что нижним пределом толщины анодного электрода можно считать $5 \mu \mathrm{m}$, так как более тонкий анодный электрод будет подвержен сильной электростатической деформации [21]. В то же время в случае выбранного диаметра рентгеновских окон $500 \mathrm{~nm}$ верхний предел толщины анода равен $10 \mu \mathrm{m}$, так как для указанного размера каналов реактивное ионное травление не позволяет достигать больших аспектных соотношений $(>20)$.

Затем вне зависимости от выбора материала электрода анода проводится осаждение $2 \mu \mathrm{m}$ борофосфороселикатного стекла (БФСС) и его оплавление в атмосфеpe $\mathrm{N}_{2}$. После осаждения БФСС не формирует сплошного слоя, имея разрывы в пленке над отверстиями. При оплавлении за счет поверхностного натяжения происходит смыкание БФСС над отверстиями и формирование сплошного слоя. При этом основной объем БФСС остается вблизи поверхности, закупоривая отверстие сверху (рис. 2,b). Далее производится плазмохимическое травление БФСС до тех пор, пока его не останется на поверхности. После этого проводится осаждение слоя мишени из Ве или $\mathrm{Si}^{*}$. Выбор материала мишени в первую очередь обусловлен необходимостью получения характеристического излучения с длиной волны в области $11.4 \mathrm{~nm}$, на которую настроена рентгенооптическая система. По этой причине отработаны технологические операции формирования тонких $\mathrm{Be}-$ и $\mathrm{Si}^{*}$-пленок, которые соответствуют указанному требованию. Затем проводится осаждение слоя $\mathrm{Si}_{3} \mathrm{~N}_{4}$ (рис. 2,c). На следующем шаге проводится напыление $\mathrm{Al}$ на обратную сторону и фотолитография с последующим совмещением по лицевой стороне. Далее на обратной стороне осуществляется травление слоев и Bosch-процесс травления кремния. При этом время травления берется таким, чтобы не 

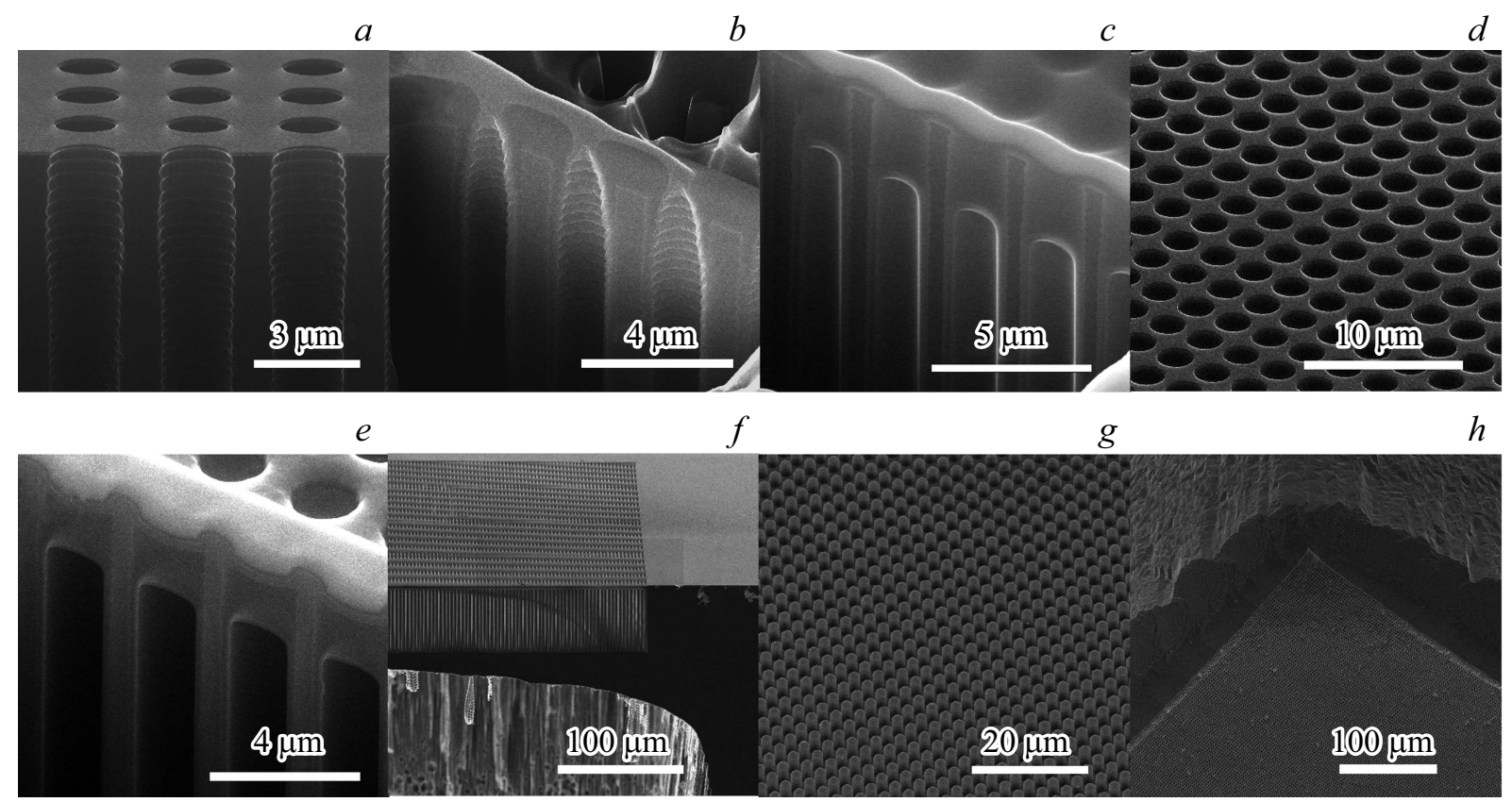

Рис. 3. Изображения матрицы анодных узлов с прострельной мишенью на различных операциях маршрута, полученные на растровом электронном микроскопе: $a$ - Bosch-процесс травления каналов; $b$ - стимулированное плазмой химическое осаждение из газовой фазы (PE CVD) БФСС; $c-$ оплавление БФСС; $d-$ плазмохимическое травление БФСС; $e-$ осаждение $\mathrm{Si}^{*}$ и $\mathrm{Si}_{3} \mathrm{~N}_{4}$; $f$ - Bosch-процесс травления с обратной стороны мембраны; $g$ - дотравливание кремния с обратной стороны в $40 \%$ растворе $\mathrm{KOH} ; h-$ удаление БФСС и $\mathrm{SiO}_{2}$ в парах концентрированной $\mathrm{HF}$ с $\mathrm{C}_{2} \mathrm{H}_{5} \mathrm{OH}$.

протравить кремниевую подложку до каналов (рис. 2,d). Следующей технологической операцией является стравливание алюминиевой маски с обратной стороны. Дотравливание кремния производится в растворе щелочи (для снижения дефектности от Bosch-процесса и лучшей контролируемости). После этого проводится стравливание нитрида с лицевой стороны. Затем производится вытравливание $\mathrm{SiO}_{2}$ и БФСС из каналов в парах $\mathrm{HF}$ и плазмохимическим травлением. В результате проводимых технологических операций получаются тонкие мембраны, имеющие электрический контакт с подложкой, висящие над каналами (рис. 2,e). На рис. 3 представлены изображения структур матрицы анодных узлов с прострельной мишенью на отдельных технологических этапах изготовления, полученные, согласно выбранному технологическому маршруту, с применением растрового электронного микроскопа.

\section{4. Результаты моделирования}

Как уже говорилось выше, матрица анодных узлов представляет собой бериллиевую прострельную мишень и электрод анода с матрицей $300 \times 300$ рентгеновских окон. Матрица анодных узлов выполнена в форме круглой мембраны радиусом $750 \mu \mathrm{m}$. Чтобы понять общие зависимости, модель была упрощена (сокращено количество ячеек в матрице рентгеновских окон). Оценено влияние типа материала и толщины анодного электрода на максимальную температуру разогрева матрицы анодных узлов. Также представлена зависимость максимальной температуры от толщины прострельной мишени. Данные оценки приведены как для краткосрочных промежутков (время экспонирования фоторентгенорезиста), так и для всей операции экспонирования пластины (несколько часов).

Физико-математическое моделирование производилось в программном пакете COMSOL Multiphysics [28]. Для моделирования был выбран модуль „Hеat Transfer in Solids“, который описывает теплопередачу в твердых телах с помощью закона теплопроводности и уравнений теплового баланса. Для описания джоулева разогрева матрицы анодных узлов автоэмиссионным током с матрицы АКУ было использовано граничное условие „Boundary Heat Source“, которое представляет собой член уравнения (3) $Q_{b}=P_{e} / S_{b e a m}$, отвечающий за дополнительные источники тепла. Следует отметить, что $P_{e}-$ мощность пучка электронов с отдельного катода, $S_{\text {beam }}$ - характерная площадь пучка электронов, приходящая на прострельную мишень. Рентгеновский литограф представляет более массивную конструкцию, чем матрица анодных узлов, поэтому условно можно считать, что вся остальная конструкция рентгеновского литографа имеет постоянную температуру. В связи с этим определено еще одно граничное условие „Temperature“, которое задает постоянную температуру $\left(20^{\circ} \mathrm{C}\right)$ на боковых гранях матрицы анодных узлов, которые контактируют с остальной конструкцией литографа. Поскольку внешние границы матрицы анодных 


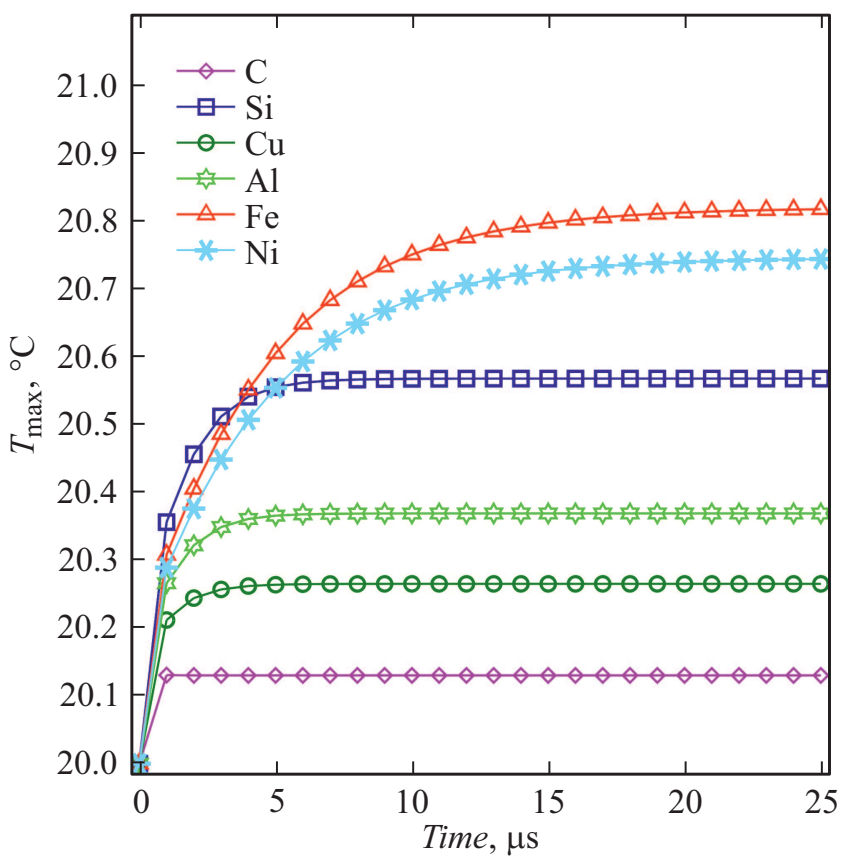

Рис. 4. Зависимость максимальной температуры матрицы анодных узлов от времени разогрева электронным током с матрицы АКУ для различных материалов электрода анода (C, Si, $\mathrm{Cu}, \mathrm{Al}, \mathrm{Fe}, \mathrm{Ni})$.

узлов контактируют с вакуумом, в процессе ее разогрева током с матрицы АКУ конвекцией и рассеиванием тепла в окружающую среду можно пренебречь. Поэтому в расчетах полагается, что на внешних границах матрицы тепловой поток равен нулю.

Важным аспектом в конструкции матрицы анодных узлов является выбор материала электрода анода (> 95\% объема матрицы анодных узлов), так как матрица анодных узлов должна обладать высокой прочностью, электростатической стабильностью, высокой теплопроводностью и теплоемкостью для быстрого отвода тепла и избегания перегрева данного узла. В работе [21] описаны способы и рекомендации для улучшения прочности и электростатической стабильности матрицы анодных узлов, поэтому в настоящей работе будет подробно рассмотрены способы снижения разогрева матрицы анодных узлов. На рис. 4 представлена зависимость максимальной температуры матрицы анодных узлов от времени воздействия электронного тока на прострельную мишень для материалов (C - алмазоподобные пленки, $\mathrm{Si}, \mathrm{Cu}, \mathrm{Al}, \mathrm{Fe}, \mathrm{Ni})$ часто использующихся в микроэлектронике. Для упрощения расчетов была промоделирована матрица анодных узлов размером $10 \times 10$ элементов, при этом толщина электрода анода составляла $5 \mu \mathrm{m}$, толщина прострельной мишени $-200 \mathrm{~nm}$, радиус матрицы анодных узлов $-25 \mu \mathrm{m}$. Из рис. 4 видно, что в течение времени экспонирования чувствительного рентгенорезиста $(25 \mu \mathrm{s})$ максимальный разогрев матрицы анодных узлов не превышает $1^{\circ} \mathrm{C}$ для лю- бого из приведенных материалов. Меньшему разогреву были подвергнуты матрицы анодных узлов с материалом анодного электрода из $\mathrm{C}, \mathrm{Cu}$ и $\mathrm{Al}$, так как они обладают большей теплопроводностью, чем остальные материалы. Еще одной особенностью данного графика является то, что для электрода анода из $\mathrm{C}, \mathrm{Cu}, \mathrm{Si}$ и $\mathrm{Al}$ максимальная температура матрицы анодных узлов быстро (в течение нескольких $\mu \mathrm{s}$ ) выходит на полку, тогда как для $\mathrm{Ni}$ и $\mathrm{Fe}$ максимальная температура продолжает расти на протяжении всего времени.

После выбора материала матрицы анодных узлов наиболее очевидным методом снижения разогрева матрицы анодных узлов представляется определение оптимальной толщины электрода анода. Уменьшение толщины электрода анода может привести к электростатической деформации и в последствии к слипанию матрицы анодных узлов и сетки (управляющего электрода), что было описано в статье [21]. На рис. 5 показана зависимость максимальной температуры матрицы анодных узлов при воздействии электронного тока с матрицы АКУ на прострельную мишень в течение $10 \mu \mathrm{s}$ от толщины электрода анода. Геометрические параметры матрицы анодных узлов оставались такими же, как описано выше. Зависимости на рис. 5 показывают, что увеличение толщины электрода анода приводит к снижению максимальной температуры разогрева матрицы анодных узлов для всех исследуемых материалов (C, $\mathrm{Si}, \mathrm{Cu}, \mathrm{Al}, \mathrm{Fe}, \mathrm{Ni}$ ). Следует отметить, что алмазоподобные пленки обладают наилучшим по сравнению с остальными материалами теплоотводом, так как максимальная температура разогрева матрицы анодных узлов практически не изме-

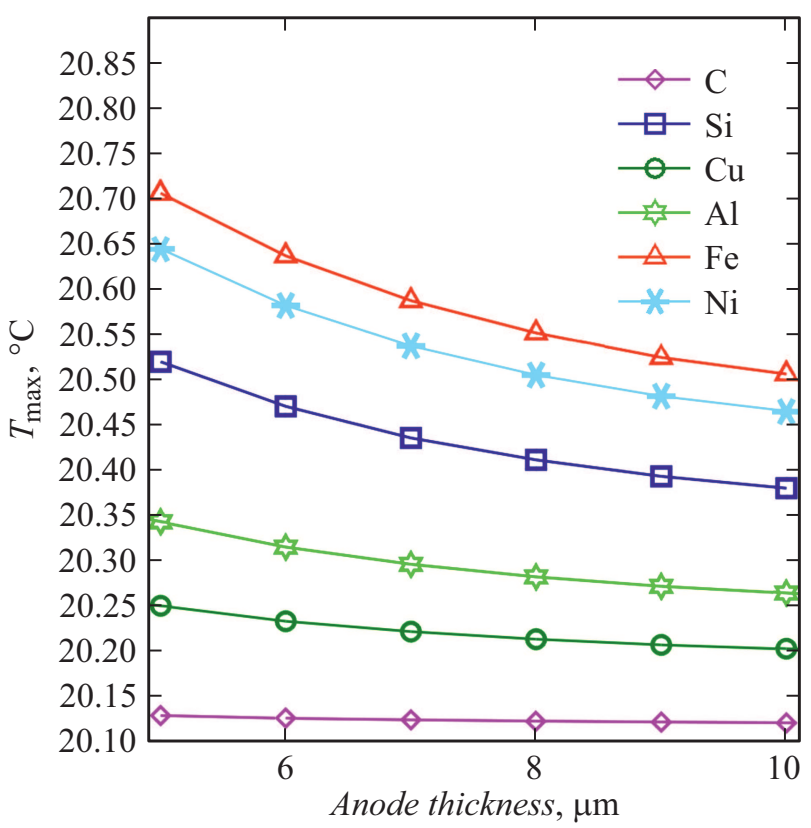

Рис. 5. Зависимость максимальной температуры матрицы анодных узлов от толщины электрода анода вследствие разогрева электронным током с матрицы АКУ в течение $10 \mu \mathrm{s}$ для различных материалов электрода анода $(\mathrm{C}, \mathrm{Si}, \mathrm{Cu}, \mathrm{Al}, \mathrm{Fe}, \mathrm{Ni})$. 


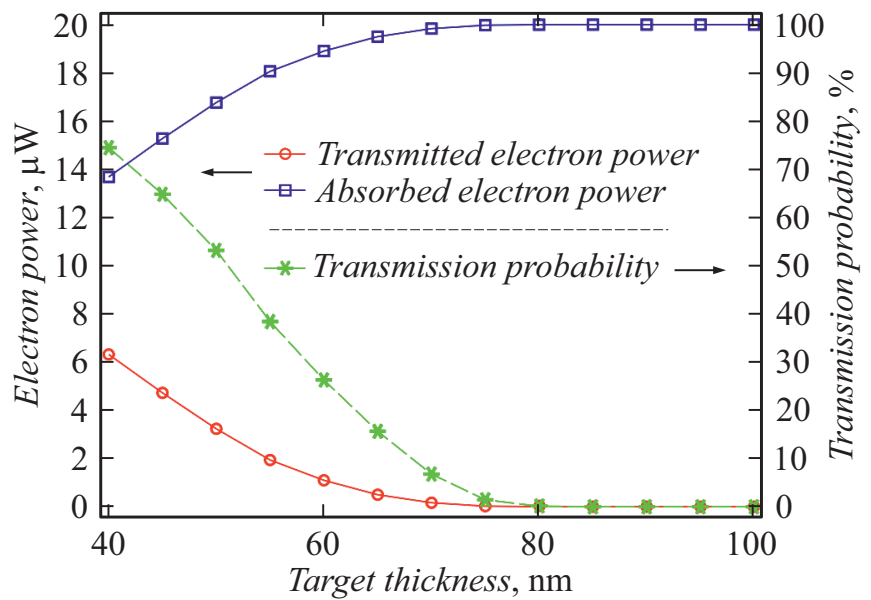

Рис. 6. Зависимость мощности, поглощенной прострельной мишенью, и доли прошедших сквозь мишень электронов от толщины бериллиевой прострельной мишени.

нялась. Поскольку оптимальный угол раствора (наклон стенки) рентгеновских окон должен быть равным $2.5^{\circ}$, что связано с требованиями к проекционному объективу (числовая апертура $\mathrm{NA}=0.4$, увеличение $10 \times$ ) для заданного размера рентгеновских окон $(\mathrm{R}=250 \mathrm{~nm})$ и шага матрицы (расстояние между окнами $3 \mu \mathrm{m}$ ), толщина анодного электрода не может быть более $25 \mu \mathrm{m}$. Это связано с тем, что при большей толщине анода происходит смыкание рентгеновских окон, либо разделяющая их стенка становится крайне тонкой (расстояние между границами окон менее $300 \mathrm{~nm}$ ).

Из работы [24] следует, что коэффициент конверсии заметно варьируется с толщиной бериллиевой прострельной мишени. В настоящей работе также показано, что максимум коэффициента конверсии энергии электронов величиной $2 \mathrm{keV}$ достигается при толщине мишени, равной $40 \mathrm{~nm}$. Однако, помимо коэффициента конверсии, в процессе работы рентгеновского нанолитографа не менее важным параметром является максимальная температура разогрева матрицы анодных узлов. Матрица анодных узлов разогревается за счет джоулева тепла от прострельной мишени, которое генерируется при воздействии на мишень автоэмиссионного тока с матрицы АКУ. Проведенные нами расчеты показывают, что область максимальной температуры разогрева матрицы анодных узлов находится непосредственно на нижней грани прострельной мишени (в ее центре).

Был произведен расчет, по результатам которого получена зависимость доли прошедших электронов от толщины прострельной мишени для тонких $(<100 \mathrm{~nm})$ бериллиевых пленок (рис. 6). Согласно нашим расчетам, доля прошедших электронов для мишени толщиной $40 \mathrm{~nm}$ составляет около $75 \%$. Необходимо отметить, что вылетающие из мишени электроны в процессе ее прохождения все равно отдают часть своей кинетической энергии атомам кристаллической решетки. Чтобы опре- делить энергию, поглощенную прострельной мишенью, необходимо знать кинетическую энергию каждого прошедшего электрона. Предложена формула для расчета мощности, поглощенной прострельной мишенью:

$$
P_{a b s}=P_{e}-\sum_{i=1}^{n} \varepsilon_{i} / t_{i},
$$

где $\varepsilon_{i}$ - энергия $i$-го электрона, прошедшего через мишень, $t_{i}$ - время прохождения $i$-го электрона через прострельную мишень. Согласно данной формуле, построена зависимость мощности, поглощенной прострельной мишенью, от толщины бериллиевой прострельной мишени (рис. 6). Несмотря на большую долю прошедших через прострельную мишень $(h=40 \mathrm{~nm})$ электронов (около $75 \%$ ), в среднем они потеряли около $59 \%$ энергии при соударениях в атомах мишени. В связи с этим доля поглощенной энергии составляет около $69 \%$ от начальной (до столкновения с мишенью) или $13.8 \mu \mathrm{W}$ с каждого АКУ. Данное значение будет использовано при расчетах максимальной температуры разогрева анодного узла. При достижении толщины прострельной мишени более $80 \mathrm{~nm}$ все электроны поглощаются прострельной мишенью.

Далее было выполнено моделирование, по результатам которого построена зависимость максимальной температуры разогрева матрицы анодных узлов от толщины бериллиевой прострельной мишени с учетом поглощенной мишенью мощности для материалов анодного электрода $\mathrm{C}$ и $\mathrm{Si}$ (см. рис. 7). Моделирование проводилось для матрицы анодных узлов размером $10 \times 10$ элементов, толщина электрода анода составляла $5 \mu \mathrm{m}$, радиус матрицы анодных узлов $-25 \mu \mathrm{m}$, разогрев электронным током производился в течение $25 \mu \mathrm{s}$. На рис. 8 приведено

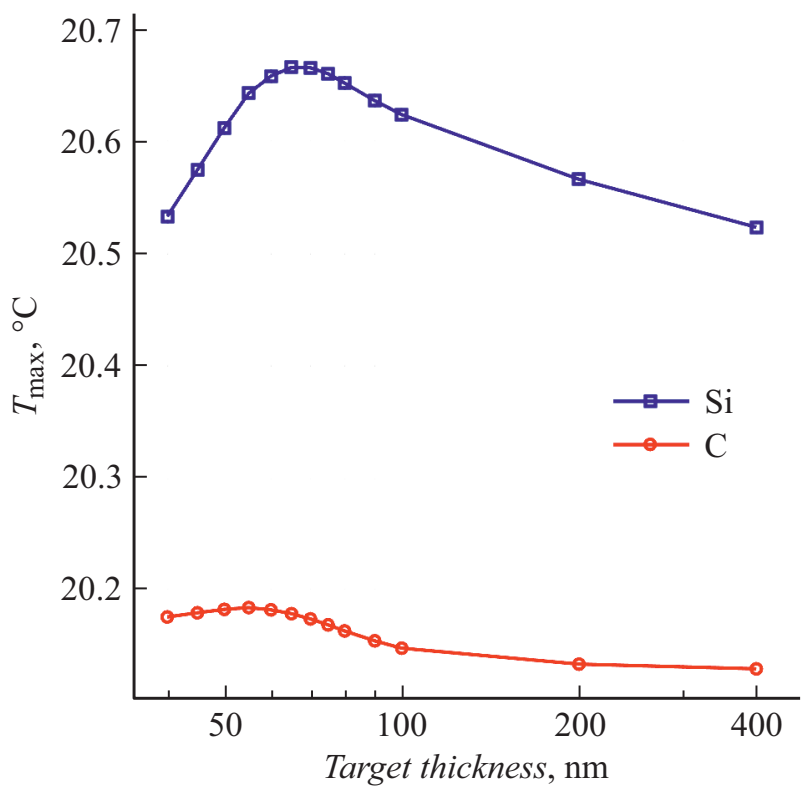

Рис. 7. Зависимость максимальной температуры матрицы анодных узлов от толщины прострельной мишени вследствие разогрева электронным током с матрицы АКУ в течение $25 \mu \mathrm{s}$. 


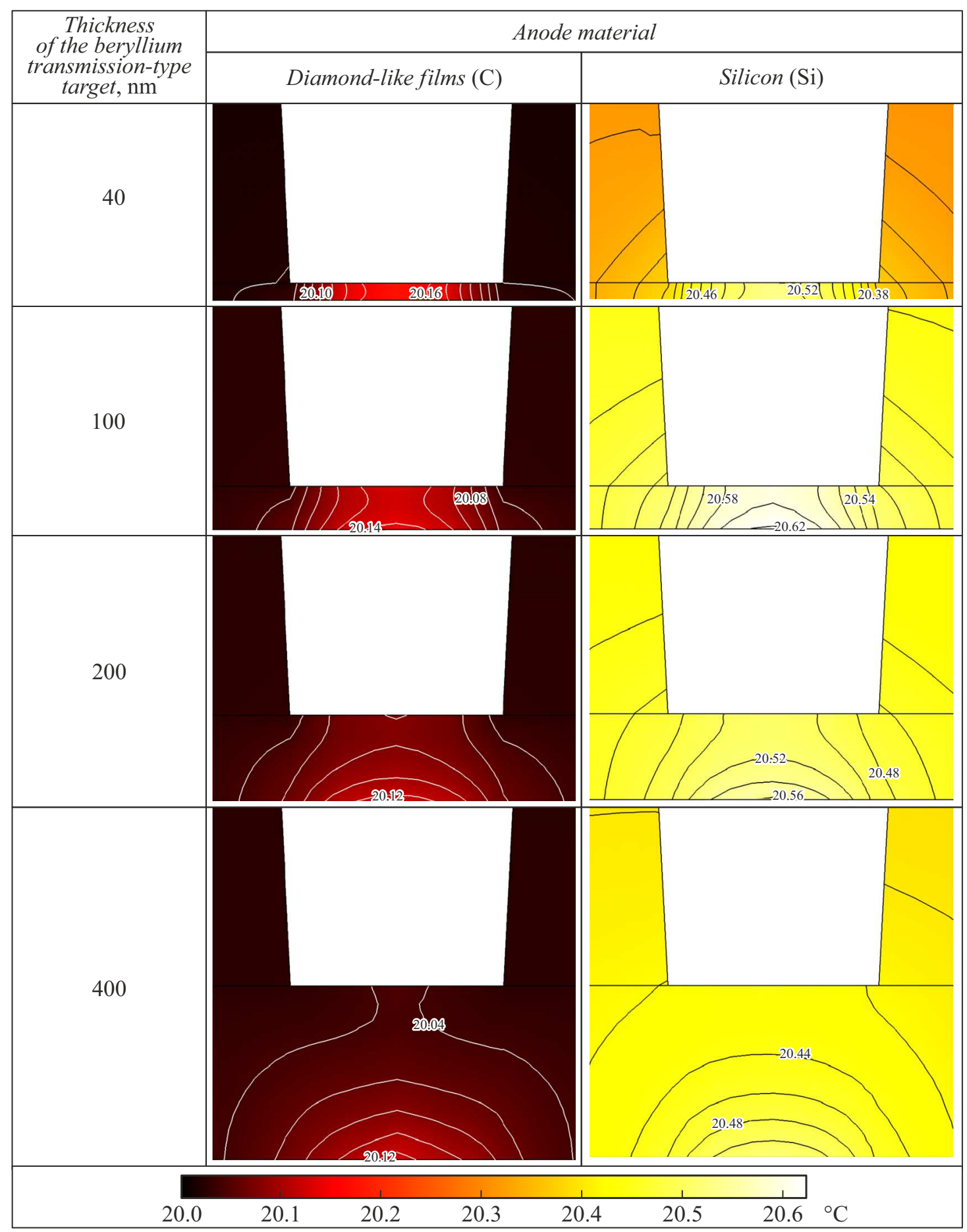

Рис. 8. Срез структуры с тепловым распределением, полученным в результате разогрева электронным током с матрицы АКУ в течение $25 \mu \mathrm{s}$, для различных толщин бериллиевой прострельной мишени $(40-400 \mathrm{~nm})$ для анодного электрода из С и $\mathrm{Si}$.

распределение температуры матрицы анодных узлов от толщины прострельной мишени вследствие разогрева электронным током с матрицы АКУ. Из рис. 7 видно, что для матрицы анодных узлов, материалом анодного электрода которой является кремний, максимальная температура разогрева достигается при толщине прострельной мишени $65 \mathrm{~nm}$. Данный эффект можно объяснить тем, что при толщине бериллиевой прострельной мишени менее $70 \mathrm{~nm}$ начинается резкое падение поглощенной мишенью мощности (рис. 7). В свою очередь, для матрицы анодных узлов материалом анодного электрода является алмазоподобная пленка максимальная температура разогрева достигается при толщине прострельной мишени $55 \mathrm{~nm}$. В отличие от матрицы анодных узлов с Si 


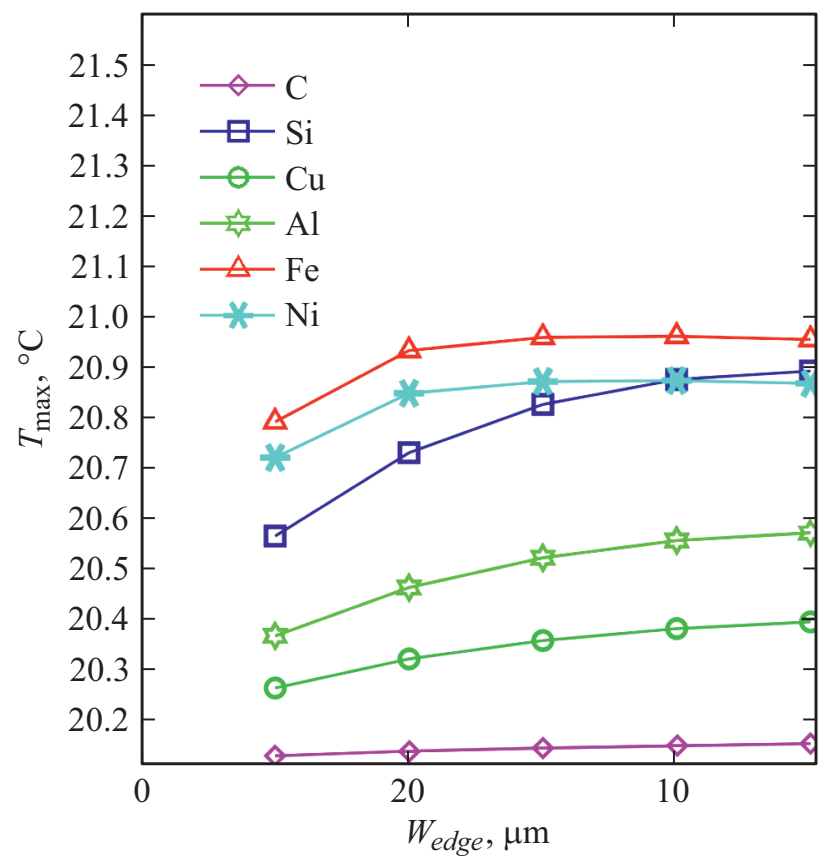

Рис. 9. Зависимость максимальной температуры матрицы анодных узлов при воздействии на нее электронного тока с матрицы АКУ от величины зазора $W_{\text {edge }}$.

анодным электродом, экстремум температуры для матрицы анодных узлов с C анодным электродом наблюдается при меньшей толщине прострельной мишени из-за высокой теплопроводности $\mathrm{C}\left(k=2500 \mathrm{~W} \cdot \mathrm{m}^{-1} \cdot \mathrm{K}^{-1}\right)$. Следует отметить, что важную роль в разогреве матрицы анодных узлов играет соотношение площади боковой поверхности (пропорционально произведению толщины на диаметр) прострельной мишени и площади соприкосновения прострельной мишени и анодного электрода.

Матрица рентгеновских окон на виде сверху представляет собой квадрат со стороной $N k$, где $N-$ размерность матрицы, $k=3 \mu \mathrm{m}-$ шаг матрицы. В свою очередь матрица анодных узлов представляет собой круглую мембрану радиуса $R$. От края матрицы рентгеновских окон до края мембраны остается некий зазор, равный $W_{\text {edge }}=R-(N k / 2)$. Для матрицы $10 \times 10$ была промоделирована зависимость максимальной температуры матрицы анодных узлов при воздействии на нее электронного тока с матрицы АКУ от величины зазора $W_{\text {edge }}$ (рис. 9). Толщина электрода анода и прострельной мишени составляла $5 \mu \mathrm{m}$ и $200 \mathrm{~nm}$ соответственно, разогрев электронным током производился в течение $10 \mu \mathrm{s}$. Материалы электрода анода из C, $\mathrm{Si}, \mathrm{Cu}$ и $\mathrm{Al}$ показали рост максимальной температуры разогрева матрицы анодных узлов при увеличении величины зазора $W_{\text {edge }}$, тогда как для $\mathrm{Ni}$ и $\mathrm{Fe}$ наблюдался рост до $W_{\text {edge }}=40 \mu \mathrm{m}$, а потом - выход на полочку максимальной температуры разогрева матрицы анодных узлов. Данный эффект объясняется тем, что матрица анодных узлов с материалом анода из $\mathrm{Ni}$ и $\mathrm{Fe}$ приходит в тепловое равновесие, когда $W_{\text {edge }}=40 \mu \mathrm{m}$.

Из рис. 4 и 7 видно, что для большинства промоделированных материалов электрода анода максимальная температура разогрева матрицы анодных узлов выходит на „полку“ (достигает максимума) в течение короткого промежутка времени $(<10 \mu \mathrm{s})$. Данный эффект можно объяснить тем, что граничное условие постоянной температуры для боковых граней мембраны сдерживает дальнейший рост температуры матрицы анодных узлов, так как радиус мембраны для матрицы рентгеновских окон $10 \times 10$ элементов составляет всего $25 \mu \mathrm{m}$. Реальная структура матрицы анодных узлов предполагает мембрану радиусом $750 \mu \mathrm{m}$ и толщиной $5-10 \mu \mathrm{m}$. На рис. 10 представлена зависимость максимальной температуры разогрева матрицы анодных узлов при воздействии электронного тока с матрицы АКУ на него от размерности матрицы рентгеновских окон (количества центров разогрева). При моделировании были заданы следующие параметры: радиус мембраны $750 \mu \mathrm{m}$; толщина электрода анода $5 \mu \mathrm{m}$; материал электрода анода $\mathrm{Si}$ или $\mathrm{C}$; толщина прострельной мишени $200 \mathrm{~nm}$; расчетное время экспонирования рентгенорезиста $10^{4} \mathrm{~s}$ (что соответствует времени обработки $150 \mathrm{~mm}$ пластины).

Также на рис. 10 представлены экстраполяционные кривые, которые позволяют оценить максимальную температуру разогрева для больших матриц вплоть до матрицы $300 \times 300$ элементов. Экстраполяционные кривые

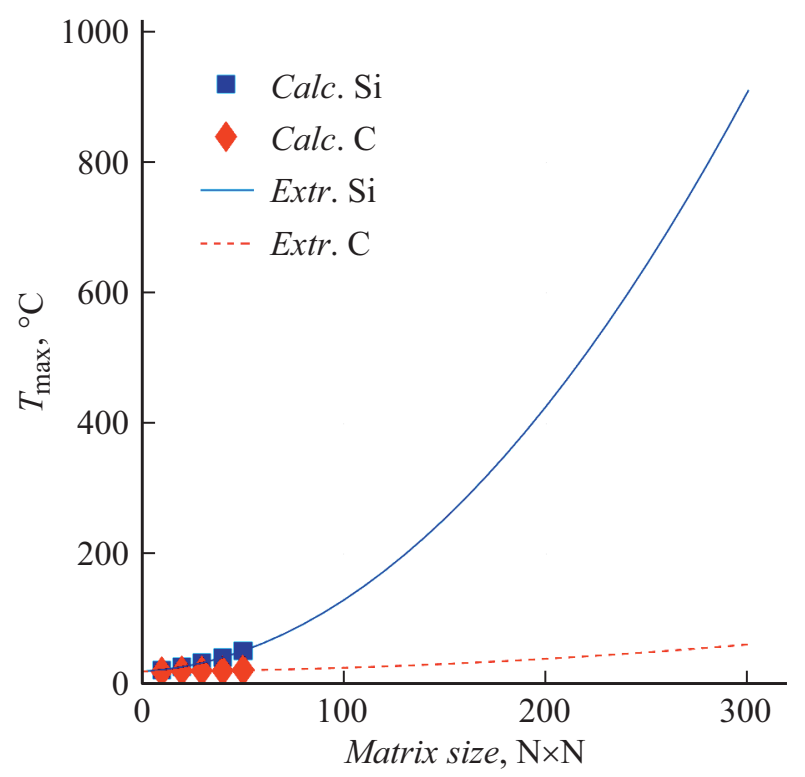

Рис. 10. Зависимость максимальной температуры разогрева матрицы анодных узлов от размера матрицы. Квадратные маркеры - вычисленные значения для матрицы анодных узлов с материалом электрода анода из $\mathrm{Si}$; ромбовидные маркеры - вычисленные значения для матрицы анодных узлов с материалом электрода анода из $\mathrm{C}$; непрерывная линия экстраполяционная кривая для материала электрода анода из $\mathrm{Si}$; пунктирная линия - экстраполяционная кривая для материала электрода анода из С. Радиус мембраны $750 \mu \mathrm{m}$. 


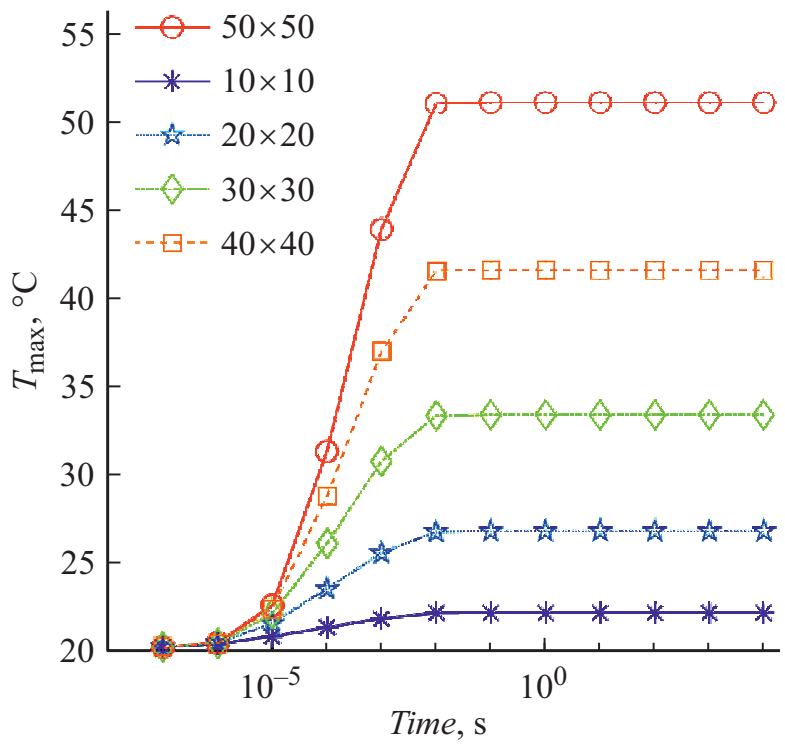

Рис. 11. Зависимость максимальной температуры разогрева матрицы анодных узлов от времени воздействия электронного тока с матрицы АКУ для различных размеров матрицы. Материал электрода анода - Si. Радиус мембраны $750 \mu \mathrm{m}$.

были построены на основе вычисленных данных для матриц $10 \times 10,20 \times 20,30 \times 30,40 \times 40$ и $50 \times 50$ с помощью программного пакета MATLAB [29]. Получены следующие уравнения для экстраполяционных кривых, которые можно использовать для оценки разогрева матрицы анодных узлов любой размерности: для $\mathrm{Si}-y=0.009399 x^{2}+0.1539 x+20$; для $\mathrm{C}-$ $y=0.000408 x^{2}+0.01577 x+20$. Из рис. 10 видно, что максимальная температура разогрева матрицы анодных узлов для материала электрода анода из $\mathrm{Si}$ составляет $>900^{\circ} \mathrm{C}$, тогда как для материала электрода анода из алмазоподобной пленки $<65^{\circ} \mathrm{C}$. Очевидно, что наиболее оптимальным материалом для электрода анода с точки зрения снижения разогрева анодного узла в целом является C.

На рис. 11 показана динамика роста максимальной температуры матрицы анодных узлов для матриц различной размерности. Исследуемая модель имела следующие параметры: радиус мембраны $750 \mu \mathrm{m}$; толщина электрода анода $5 \mu \mathrm{m}$; материал электрода анода $\mathrm{Si}$; толщина прострельной мишени $200 \mathrm{~nm}$. Данные моделирования показывают, что максимальная температура разогрева матрицы анодных узлов достигает предельных значений в течение сотых долей секунды.

\section{Заключение}

Подводя итоги всего вышесказанного, необходимо сказать, что важным аспектом правильной и точной работы рентгеновского нанолитографа является температурная стабилизация как всего устройства, так и матрицы анодных узлов в частности. Причиной неисправной работы рентгеновского нанолитографа может служить термическое расширение и деградация матрицы анодных узлов вследствие ее значительного разогрева под воздействием электронного тока с матрицы АКУ на прострельную мишень. Одним из методов снижения разогрева матрицы анодных узлов, рассмотренных в настоящей работе, является выбор наиболее теплопроводного и теплоемкого материала анодных узлов. На основании разработанной физико-математической модели был произведен расчет разогрева матрицы анодных узлов для различных материалов электрода анода (анодной мембраны), среди которых наиболее оптимальным материалом для электрода анода с точки зрения снижения разогрева анодного узла в целом оказались алмазоподобные пленки. Экстраполяция рассчитанных зависимостей температуры разогрева от размера матрицы показала, что для матрицы анодных узлов размером $300 \times 300$ элементов максимальная температура разогрева для электрода анода из алмазоподобной пленки составляет не более $65^{\circ} \mathrm{C}(\Delta T=42 \mathrm{~K})$.

Предложены методы оптимизации конструкции матрицы анодных узлов для снижения ее разогрева и повышения теплоотвода с нее: увеличение толщины электрода анода и прострельной мишени. Расчеты показали, что увеличение толщины электрода анода в 2 раза (c 5 до $10 \mu \mathrm{m}$ ) приводит к снижению максимальной температуры разогрева матрицы анодных узлов от 5 до $30 \%$ в зависимости от материала электрода анода. Также произведены расчеты для различной толщины $(40-400 \mathrm{~nm})$ прострельной мишени с учетом поглощенной мишенью мощности для материалов электрода анода С и $\mathrm{Si}$, в результате которых было выявлено, что экстремум максимальной температуры разогрева достигается при толщине прострельной мишени 55 и $65 \mathrm{~nm}$ соответственно. Данный эффект связан с тем, что при толщине бериллиевой прострельной мишени менее $70 \mathrm{~nm}$ начинается резкое падение поглощенной мишенью мощности (рис. 7). Необходимо сказать, что увеличение толщины прострельной мишени приводит к снижению коэффициента конверсии энергии электронов в энергию рентгеновского излучения. Оптимальным диапазоном толщин прострельной мишени с точки зрения компромисса между коэффициентом конверсии и тепловой нагрузкой на матрицу анодных узлов с алмазоподобным анодным электродом можно считать диапазон $100-200 \mathrm{~nm}$ для энергии электронов $2 \mathrm{keV}$. Несмотря на то что в данном диапазоне коэффициент конверсии снижается на 10-20\% относительно коэффициента конверсии при выборе оптимальной толщины мишени, равной $40 \mathrm{~nm}$ [24], тем не менее максимальная температура разогрева матрицы анодных узлов уменышается на 20-25\%. В свою очередь, для матрицы анодных узлов с кремниевым анодным электродом наиболее оптимальным вариантом в данном случае представляется бериллиевая прострельная мишень толщиной $40 \mathrm{~nm}$.

Таким образом, сформированные в ходе работы рекомендации, направленные на снижение эффекта теп- 
лового разогрева матрицы анодных узлов в составе источников мягкого рентгеновского излучения, могут играть важную роль для повышения стабильности и надежности процессов рентгеновской нанолитографии на их основе. Результаты моделирования также могут быть применимы для разработки и конструирования миниатюрных устройств генерации рентгеновского излучения.

\section{Финансирование работы}

Работа была выполнена с использованием оборудования ЦКП „МСТ и ЭКБ“ (МИЭТ) при финансовой поддержке Министерства науки и высшего образования Российской Федерации (№ 075-03-2020-216, 0719-20200017, мнемокод FSMR-2020-0017).

\section{Конфликт интересов}

Авторы заявляют, что у них нет конфликта интересов.

\section{Список литературы}

[1] T. Matsui. J. Infrared Milli Terahz Waves, 38 (9), 1140 (2017). DOI: $10.1007 / \mathrm{s} 10762-017-0387-9$

[2] J. Feng, X. Li, J. Hu, J. Cai. J Electromagn. Eng Sci., 20 (1), 1 (2020). DOI: 10.26866/jees.2020.20.1.1

[3] B. Levush. IVEC (Busan, South Korea, 2019), p. 1-5. DOI: 10.1109/IVEC.2019.8745196

[4] S.A. Guerrera, A.I. Akinwande. Nanotech., 27, 295302 (2016). DOI: 10.1088/0957-4484/27/29/295302

[5] J.-W. Han, M.-L. Seol, D.-I. Moon, G. Hunter, M. Meyyappan. Nat. Electron., 2, 405 (2019). DOI: $10.1038 / \mathrm{s} 41928-019-0289-\mathrm{z}$

[6] M. Liu, T. Li, Y. Wang. J. Vac. Sci. Technol. B, 35, 031801 (2017). DOI: $10.1116 / 1.4979049$

[7] Y. Huang, Z. Deng, W. Wang, C. Liang, J. She, S. Deng, N. Xu. Sci. Rep., 5, 10631 (2015). DOI: 10.1038/srep10631

[8] P. Zhang, Y.Y. Lau. J. Plasma Phys., 82, 595820505 (2016). DOI: $10.1017 / \mathrm{S} 002237781600091 \mathrm{X}$

[9] W.-T. Chang, H.-J. Hsu, P.-H. Pao. Micromachines, 10, 858 (2019). DOI: $10.3390 / \mathrm{mi} 10120858$

[10] J.-W. Han, D.-I. Moon, M. Meyyappan. Nano Lett., 17, 2146 (2017). DOI: 10.1021/acs.nanolett.6b04363

[11] J. Xu, Z. Gu, W. Yang, Q. Wang, X. Zhang, Nanoscale Res. Lett., 13, 311 (2018.) DOI: 10.1186/s11671-018-2736-6

[12] M. Liu, Y. Lei, Y. Yang, T. Li, Y. Wang. Proc. 2019 International Conference on Manipulation, Automation and Robotics at Small Scales (Helsinki, Finland, 2019), 1. DOI: $10.1109 /$ marss.2019.8860991

[13] N.A. Djuzhev, G.D. Demin, T.A. Gryazneva, V.Yu. Kireev, D.V. Novikov. Proc. 2018 IEEE Conference of Russian Young Researchers in Electrical and Electronic Engineering (IEEE, Moscow, Russia, 2018) DOI: 10.1109/EIConRus.2018.8317498

[14] R. Menon, A. Patel, D. Gil, H.I. Smith. Mater. Today, 8 (2), 26 (2005)

[15] Г.В. Белокопытов, Ю.В. Рыжикова. Микроэлектроника, 40 (6), 453 (2011)
[16] U. Dauderstädt, P. Askebjer, P. Björnängen, P. Dürr, M. Friedrichs, M. List, D. Rudloff, J.-U. Schmidt, M. Müller, M. Wagner. Proc. SPIE, 7208, 720804 (2009).

[17] Электронный ресурс. Режим доступа: https://heidelberginstruments.com/en/products/dwl-66.html

[18] Н.А. Дюжев, Г.Д. Демин, Н.А. Филиппов, И.Д. Евсиков, П.Ю. Глаголев, М.А. Махиборода, Н.И. Чхало, Н.Н. Салащенко, С.В. Филиппов, А.Г. Колосько, E.O. Попов, В.А. Беспалов. ЖТФ, $89(12), 1836$ (2019) DOI: $\quad 10.21883 /$ JTF.2019.12.48479.137-19 [N.A. Djuzhev, G.D. Demin, N.A. Filippov, I.D. Evsikov, P.Y. Glagolev, M.A. Makhiboroda, N.I. Chkhalo, N.N. Salashchenko, S.V. Filippov, A.G. Kolosko, E.O. Popov, V.A. Bespalov. Tech. Phys., 64 (12), 1742 (2019). DOI: $10.1134 / \mathrm{S} 1063784219120053]$

[19] N.N. Salashchenko, N.I. Chkhalo, N.A. Djuzhev. J. Surf. Invest.: X-Ray, Synchrotron Neutron Tech., 12, 944 (2018). DOI: $10.1134 / \mathrm{S} 1027451018050324$

[20] G.D. Demin, N.A. Djuzhev, N.A. Filippov, P.Yu. Glagolev, I.D. Evsikov, N.N. Patyukov. J. Vac. Sci. Technol. B, 37, 022903 (2019). DOI: 10.1116/1.5068688

[21] П.Ю. Глаголев, Г.Д. Демин, Г.И. Орешкин, Н.И. Чхало, Н.А. Дюжев. ЖТФ, 90 (11), 1789 (2019). DOI: 10.21883/JTF.2020.11.49964.137-20 [P.Yu. Glagolev, G.D. Demin, G.I. Oreshkin, N.I. Chkhalo, N.A. Djuzhev. Tech. Phys., 65 (11), 1709 (2020). DOI: $10.1134 / \mathrm{S} 1063784220110122]$

[22] V.P. Nazmov, E.F. Reznikova, A. Somogyi, J. Mohr, V. Saile. Optical Science and Technology, the SPIE 49th Annual Meeting (Denver, Colorado, United States, 2004), p. 235. DOI: $10.1117 / 12.562615$

[23] G.D. Demin, N.A. Dyuzhev, M.A. Makhiboroda, A.Y. Lopatin, N.I. Chkhalo, A.E. Pestov, N.N. Salashchenko. International Conference on Micro- and Nano-Electronics. 2018. (Zvenigorod, Russian Federation: SPIE, 2019), p. 67. DOI: $10.1117 / 12.2522105$

[24] A.Ya. Lopatin, D.E. Par'ev, A.E. Pestov, N.N. Salashchenko, N.I. Chkhalo, G.D. Demin, N.A. Dyuzhev, M.A. Makhiboroda, A.A. Kochetkov. J. Exp. Theor. Phys. 127 (6), 985 (2018). DOI: 10.1134/S1063776118100175

[25] N.A. Dyuzhev, G.D. Demin, T.A. Gryazneva, A.E. Pestov, N.N. Salashchenko, N.I. Chkhalo, F.A. Pudonin. Kratk. Soobshch. Fiz. FIAN, 12, 56 (2017).

[26] C. Montcalm, S. Bajt, P.B. Mirkarimi, E.A. Spiller, F.J. Weber, J.A. Folta. 23rd Annual International Symposium on Microlithography (Santa Clara, CA, United States 1998), p. 46. DOI: $10.1117 / 12.309600$

[27] N.A. Dyuzhev, G.D. Demin, T.A. Gryazneva, A.E. Pestov, N.N. Salashchenko, N.I. Chkhalo, F.A. Pudonin. Bull. Lebedev Phys. Inst. 45 (1), 1 (2018). DOI: $10.3103 / \mathrm{S} 1068335618010013$

[28] COMSOL Multiphysics, COMSOL AB, Stockholm, Sweden, https://www.comsol.com/

[29] MATLAB, MathWorks, https://www.mathworks.com/products/matlab 